\title{
Chronischer Rückenschmerz
}

\section{Neuropathische Anteile erkennen und gezielt behandeln}

- Chronische Rückenschmerzen gehen in vier von zehn Fällen mit neuropathischen Schmerzanteilen einher. Die Patientenzufriedenheit mit der Schmerztherapie steht und fällt damit, dass diese Schmerzkomponente früh erkannt und spezifisch behandelt wird.

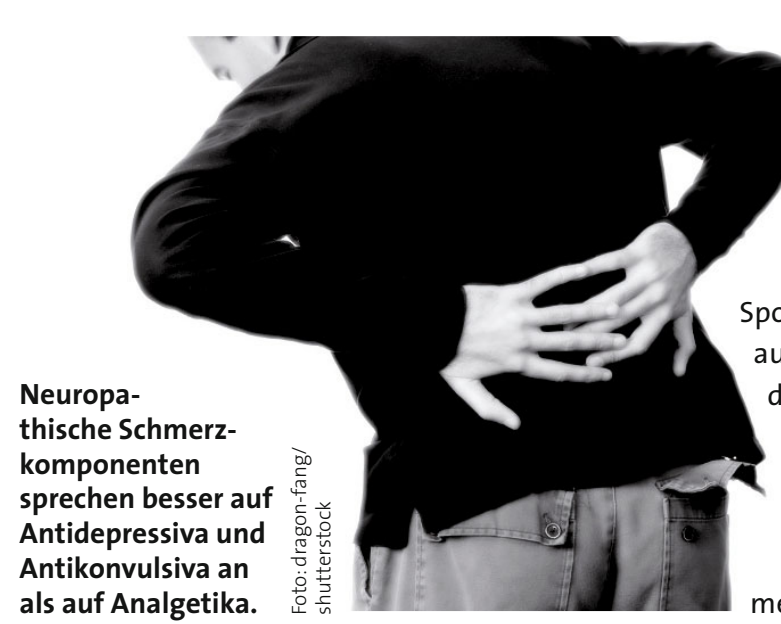

Derzeit zielt die medikamentöse Therapie bei chronischen Rückenschmerzen meist darauf, die nozizeptiven Schmerzen mit nicht steroidalen Antirheumatika oder Opioiden auszuschalten, berichtete
Prof. Ralf Baron, Universität Kiel. In vielen Fällen ist das aber zu wenig, etwa wenn im Rahmen der Degeneration in die Bandscheibe einsprießende Nervenfasern mechanisch geschädigt werden oder wenn ein im Knochen sitzender Tumor die ossären Nerven zerstört und sich so dem zunächst rein nozizeptiven Schmerz neuropathische Anteile hinzugesellen. Zu den Diagnosen, bei denen gehäuft neuropathische Schmerzen anzutreffen sind, gehören Bandscheibenvorfälle, Spondylosen und Radikulitiden, aber auch die Osteoporose und Traumata der Wirbelsäule.

Eine Fragebogenaktion mit fast 8000 Rückenschmerzpatienten im Rahmen des painDETECT-Projektes ergab, dass mehr als ein Drittel von ihnen mit über 90\%iger Wahrscheinlichkeit auch neuropathische Schmerzen hatten. Diese Patienten litten im Schnitt unter stärkeren Schmerzen als jene, die mit hoher Wahrscheinlichkeit einen rein nozizeptiven Schmerz hatten. Das pain-
DETECT-Projekt bietet übrigens einen einfachen Fragebogen an, der es auch nicht auf Schmerztherapie spezialisierten Ärzten erleichtert, den Verdacht auf eine neuropathische Schmerzkomponente rasch zu erhärten.

Wie bei Diabetes und Zoster sprechen auch neuropathische Schmerzen am Rücken besser auf Antidepressiva und Antikonvulsiva an als auf Analgetika. Unter den Antikonvulsiva sollte am neuronalen Kalziumkanal ansetzenden Wirkstoffen wie Pregabalin (Lyrica ${ }^{\circledR}$ ) der Vorzug gegeben werden, weil sie die beste Evidenz vorzuweisen haben, so die Leitlinie Neuropathischer Schmerz der Deutschen Gesellschaft für Neurologie. Für Pregabalin liegt eine offene Beobachtungsstudie beim neuropathischen Rückenschmerz mit über 4000 Patienten vor, die einen fast $60 \%$ igen Rückgang der Schmerzintensität in sechs Wochen zeigt.

\footnotetext{
- Manuela Arand

Quelle: Pressekonferenz „Schmerzen kosten nicht nur Nerven “, Deutscher Kongress für Orthopädie und Unfallchirurgie, Berlin, 21. Oktober 2009 (Veranstalter: Pfizer)
}

\section{Therapie der Enuresis nocturna}

\section{Bei Urinabgang klingelt es}

— Wenn Kinder im Alter über fünf Jahren nachts noch einnässen, sollte zunächst ihr Trink- und Miktionsverhalten überprüft werden. Es wird empfohlen, dass sie die Hauptmenge an Flüssigkeit vor $17 \mathrm{Uhr}$ trinken, zuckerhaltige Getränke (zumindest in der zweiten Tageshälfte) meiden und bei Harndrang gleich eine Toilette aufsuchen.

Der nächste Schritt ist eine apparative Verhaltenstherapie (AVT) oder Alarmtherapie: Wenn es nachts zu einem Urinabgang kommt, wird durch einen Urinsensor ein Klingelsignal ausgelöst. Das Kind wird wach und kann zur Toilette gehen, um die Blase vollständig zu entleeren. Die
Heilungsrate mit dieser Maßnahme liegt bei $75-85 \%$. Im Durchschnitt nach zwei Monaten sind die Kinder trocken.

Damit die Alarmtherapie Erfolg hat, muss das Therapiegerät einige Anforderungen erfüllen:

- Das Wecksignal darf nicht im oder am Bett abzustellen sein. Vielmehr müssen die Kinder dazu aufstehen und die Toilette aufsuchen.

- Der Urinsensor sollte großflächig sein, damit frühzeitig ein Alarmsignal ausgelöst werden kann.

- Der Urinsensor sollte möglichst komfortabel sein, um einen ruhigen Schlaf zu ermöglichen.
- Die Apparatur muss zuverlässig funktionieren.

Um den Behandlungserfolg sicherzustellen, sollte die Verordnung einer AVT, zum Beispiel mit der STERO Enurex Klingelhose $^{\circledR}$, von einer kompetenten Betreuung begleitet werden. Umstände, die den Behandlungserfolg erschweren, wie z.B. schwere Erweckbarkeit, Motivationsprobleme bei Kind und Eltern oder Fehler während der Behandlung müssen möglichst schnell erkannt werden, um einen Behandlungsabbruch zu verhindern.

- Red

Quelle: Practica, Bad Orb, November 2009; STERO Medizinische Geräte 